\title{
OPTIMIZATION OF INTERDIGITATED BACK CONTACT SILICON HETEROJUNCTION SOLAR CELLS BY TWO-DIMENSIONAL NUMERICAL SIMULATION
}

\author{
Meijun Lu, Ujjwal Das, Stuart Bowden, Steven Hegedus, and Robert Birkmire \\ Institute of Energy Conversion, University of Delaware, Newark, DE 19716 U.S.A.
}

\begin{abstract}
In this paper, two-dimensional (2D) simulation of interdigitated back contact silicon heterojunction (IBC-SHJ) solar cells is presented using Sentaurus Device, a software package of Synopsys TCAD. A model is established incorporating a distribution of trap states of amorphous-silicon material and thermionic emission across the amorphous-silicon / crystalline-silicon heterointerface. The 2D nature of IBC-SHJ device is evaluated and current density-voltage $(\mathrm{J}-\mathrm{V})$ curves are generated. Optimization of IBC-SHJ solar cells is then discussed through simulation. It is shown that the open circuit voltage $\left(\mathrm{V}_{\mathrm{OC}}\right)$ and short circuit current density $\left(\mathrm{J}_{\mathrm{SC}}\right)$ of IBC-SHJ solar cells increase with decreasing front surface recombination velocity. The $\mathrm{J}_{\mathrm{SC}}$ improves further with the increase of relative coverage of p-type emitter contacts, which is explained by the simulated and measured position dependent laser beam induced current (LBIC) line scan. The S-shaped J-V curves with low fill factor (FF) observed in experiments are also simulated, and three methods to improve FF by modifying the intrinsic a-Si buffer layer are suggested: (i) decreased thickness, (ii) increased conductivity, and (iii) reduced band gap. With all these optimizations, an efficiency of $26 \%$ for IBC-SHJ solar cells is potentially achievable.
\end{abstract}

\section{INTRODUCTION}

An interdigitated back contact silicon heterojunction (IBC-SHJ) solar cell was proposed recently [1]. The IBC-SHJ combines the advantages of the interdigitated back contact (IBC) [2] and silicon heterojunction [3] solar cells. Having all the contacts at the back of the cell eliminates contact shading, leading to a higher short-circuit current $\left(\mathrm{J}_{\mathrm{SC}}\right)$. The rear junction avoids trade-off between series resistance and reflectance, reducing the series resistance losses. It also simplifies cell stringing during module fabrication and improves the packing factor. Being a heterojunction device, IBC-SHJ also has the potential of higher open circuit voltage $\left(\mathrm{V}_{\mathrm{OC}}\right)$ due to the better surface passivation of the deposited amorphous silicon (a-Si) layer [4]. The low temperature deposition, instead of the high temperature diffusion, decreases the thermal stress and reduces the bowing. This is more beneficial for thin wafers, which is the trend of future silicon solar cells. Also, the patterning is easier in heterojunctions than the diffused junctions in traditional rear junction cells since it is much easier to mask and etch depositions than diffusions, and further isolation between $\mathrm{p} / \mathrm{n}$ a-Si layers is not always necessary.
In this work, numerical simulations of IBC-SHJ devices are presented. A 2D model is established, 2D nature of IBC-SHJ device is evaluated, and optimizations of IBC-SHJ solar cells are discussed, including the effect of front surface recombination velocity, strip widths, laser beam induced current (LBIC) line scan, and methods to improve FF for S-shaped J-V curve.

\section{TWO-DIMENSIONAL MODELLING}

The interdigitated back contact heterojunction device combines two challenging modeling requirements. Firstly, incorporating amorphous silicon with the heterojunction devices is more difficult to model than normal diffused junctions with only crystalline materials, and popular photovoltaic modeling software such as PC1D is not suitable. Secondly, an all back contact device is an inherently two-dimensional structure, which requires more complicated 2D simulation rather than 1D modeling. In this paper, we use "Sentaurus Device" [5], which is a simulator from software package Synopsys TCAD. Sentaurus Device is extensively used for modeling opto-electronic devices, and it is well suitable for modeling solar cells [6]. The latest version includes complex defect models allowing the simulation of devices which include amorphous silicon. It also allows special specification of transport physics for the hetero-interface.

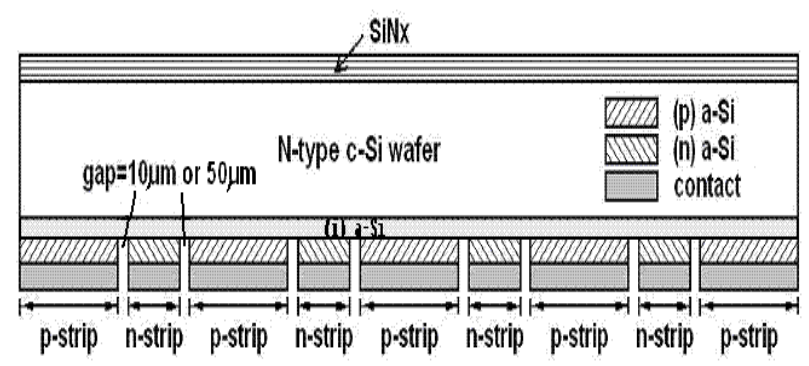

Figure 1. Schematic diagram used for the simulation of IBC-SHJ solar cells. For clarity, the dimension is exaggerated.

A schematic diagram of the structure of an IBC$\mathrm{SHJ}$ solar cell used for the numerical simulation is shown in Figure 1, and a detailed discussion of the device is given in reference [1]. A $2.5 \Omega \bullet \mathrm{cm}$ n-type c-Si wafer (300 $\mu \mathrm{m})$ is passivated with $\operatorname{SiN}_{\mathrm{x}}(75 \mathrm{~nm})$ at the front surface. At the rear of the wafer, there are interdigitated strips of $p$ and $\mathrm{n}$ a-Si:H (both $20 \mathrm{~nm}$ ), with or without the amorphous 
silicon buffer (varies between 0 to $20 \mathrm{~nm}$ ). The default lateral dimensions of $p$ - and $n-$ strips are $1.2 \mathrm{~mm}$ and 0.5 $\mathrm{mm}$ respectively, and the gap between them is set to be $10 \mu \mathrm{m}$ for cells without back surface buffer layer, and 50 $\mu \mathrm{m}$ for the one with buffer layer [7]. These dimensions will be tuned in the simulation.

The three governing semiconductor equations Poisson equations, the electron and hole continuity equations - together with drift-diffusion model in Sentaurus Device were used. The Auger and Shockley-Read-Hall recombination were also considered for crystalline silicon wafer. For a-Si:H layers, the critical parameters such as band gap, doping (conductivity), the energy distribution of the exponential band tails, and the Gaussian distribution of mid-gap trap states, are chosen based on reference [8] and are tuned to fit the measured optoelectronic properties of the deposited a-Si:H layers. The default values of them are listed in Table 1.

For c-Si/a-Si:H interfaces at the back surface, a thermionic emission model was used, in which the distribution function of the interface defect is modeled by two capture cross-sections, one for the holes and one for the electrons. An AM1.5G solar spectrum is used for the optical generation to simulate the J-V curve under standard one-sun illumination conditions at an intensity of $100 \mathrm{~mW} / \mathrm{cm}^{2}$.

\section{RESULTS}

\section{Two-dimensional distribution of current flow}

First, an IBC-SHJ solar cell with ideal conditions (without back surface buffer layer, front surface recombination velocity $S R V=10 \mathrm{~cm} / \mathrm{s}$, no interface defects for c-Si/a-Si:H interfaces at the back surface) is simulated to evaluate the two-dimensional (2D) nature of the cell. Figure 2 shows the 2D spatial distribution of the total net current flow in IBC-SHJ solar cells and electrostatic potential near back surface at short circuit with no voltage bias, where each is shown for only half of the cell due to symmetry, i.e. 2.5 p-strips and 2 n-strips. It should be noticed that the regions of the wafer shown in yscale are different, and both are exaggerated. As seen, the IBC-SHJ solar cell has a two-dimensional current flow pattern. The current mainly flows laterally from base (nfingers) to the emitter ( $p$-fingers) in the cell. Also, the IBC$\mathrm{SHJ}$ solar cell can be divided into three regions: $n$ collection region, $\mathrm{p}$ - collection region, and the diffusion region. Most of the bulk $\mathrm{c}-\mathrm{Si}$ wafer can be defined as diffusion region, where the diffusion dominates the carrier transport. While at rear surface $(\sim 1 \mu \mathrm{m})$, the $2 \mathrm{D}$ electric field is built up where drift transport is significant, and two ( $p$ - and $n-$ ) collection regions can be defined. Hence, the photogenerated carriers will mainly diffuse in the bulk of the device until they reach the back surface near depletion region $(\sim 1 \mu \mathrm{m})$ and then be separated by the electric field. Because of this, the use of long lifetime c-Si wafer is required for good collection.

Table 1. Default parameters used in the simulation: band gap, doping, and defect-state distributions.

\begin{tabular}{|c|c|c|c|c|}
\hline \multicolumn{2}{|l|}{ Material } & n-type a-Si:H & buffer a-Si:H & p-type a-Si:H \\
\hline \multicolumn{2}{|l|}{ Band gap Eg } & $1.70 \mathrm{eV}$ & $1.70 \mathrm{eV}$ & $1.65 \mathrm{eV}$ \\
\hline \multicolumn{2}{|l|}{ Doping } & $\begin{array}{l}1.51 \times 10^{19} \mathrm{~cm}^{-3} \\
\text { (phosphorus) }\end{array}$ & $\begin{array}{l}2.2 \times 10^{15} \mathrm{~cm}^{-3} \\
\text { (phosphorus) }\end{array}$ & $\begin{array}{l}2.05 \times 10^{19} \mathrm{~cm}^{-3} \\
\text { (boron) }\end{array}$ \\
\hline \multirow[t]{2}{*}{ Conduction tail states } & $N^{c \text {-tail }} A$ & $10^{21} \mathrm{~cm}^{-3}$ & $10^{18} \mathrm{~cm}^{-3}$ & $10^{21} \mathrm{~cm}^{-3}$ \\
\hline & $E^{c \text {-tail }} A$ & $0.07 \mathrm{eV}$ & $0.06 \mathrm{eV}$ & $0.07 \mathrm{eV}$ \\
\hline \multirow[t]{2}{*}{ Valence tail states } & $N_{D}^{v-\text { tail }}$ & $10^{21} \mathrm{~cm}^{-3}$ & $10^{18} \mathrm{~cm}^{-3}$ & $10^{21} \mathrm{~cm}^{-3}$ \\
\hline & $E_{D}^{v \text {-tail }}$ & $0.12 \mathrm{eV}$ & $0.09 \mathrm{eV}$ & $0.12 \mathrm{eV}$ \\
\hline \multirow{3}{*}{$\begin{array}{l}\text { Acceptor-like }(\mathrm{A}) \\
\text { dangling bond states }\end{array}$} & $N_{A}^{d b}$ & $10^{19} \mathrm{~cm}^{-3}$ & $10^{16} \mathrm{~cm}^{-3}$ & $10^{19} \mathrm{~cm}^{-3}$ \\
\hline & $E_{A}^{d b}$ & $0.7 \mathrm{eV}$ & $1.1 \mathrm{eV}$ & $1.3 \mathrm{eV}$ \\
\hline & $\sigma_{A}^{d b}$ & $0.2 \mathrm{eV}$ & $0.15 \mathrm{eV}$ & $0.2 \mathrm{eV}$ \\
\hline \multirow{3}{*}{$\begin{array}{l}\text { Donor-like }(\mathrm{D}) \text { dangling } \\
\text { bond states }\end{array}$} & $N^{d b}{ }_{D}$ & $10^{19} \mathrm{~cm}^{-3}$ & $10^{16} \mathrm{~cm}^{-3}$ & $10^{19} \mathrm{~cm}^{-3}$ \\
\hline & $E^{d b}{ }_{D}$ & $0.45 \mathrm{eV}$ & $0.9 \mathrm{eV}$ & $1.1 \mathrm{eV}$ \\
\hline & $\sigma^{d b}{ }_{D}$ & $0.2 \mathrm{eV}$ & $0.15 \mathrm{eV}$ & $0.2 \mathrm{eV}$ \\
\hline
\end{tabular}




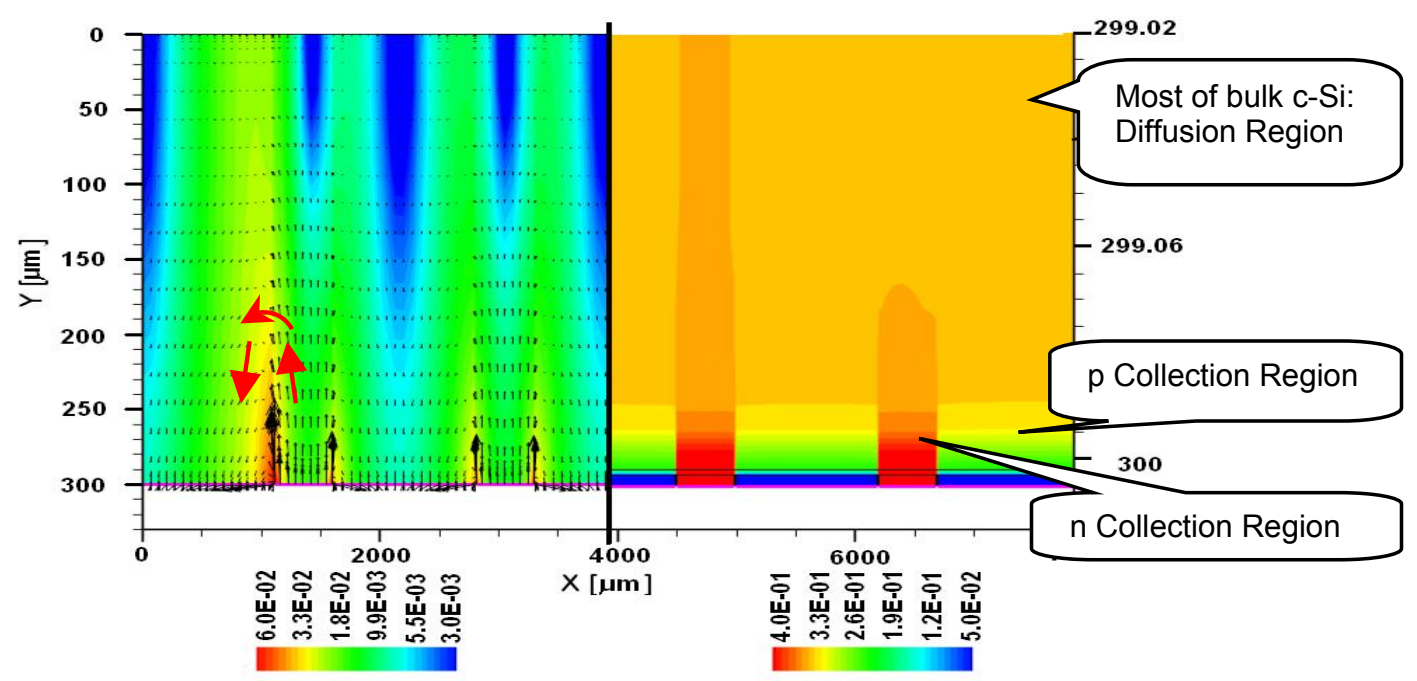

Figure 2. Total net current flow (left) in $300 \mu \mathrm{m}$ thick IBC-SHJ (arrow indicating current flow) and electrostatic potential (right) within $1 \mu \mathrm{m}$ of the back surface.

\section{Effect of front surface passivation}

Simulations with different front SRV were performed and the resulting $\mathrm{J}-\mathrm{V}$ curves are shown in Figure 3, where the back surface is assumed as ideal. As seen, both $J_{S c}$ and $V_{O c}$ increase as SRV decreases, resulting in higher cell efficiency. Since most carriers are generated near the front surface, while the $p-n$ junction is far at back surface, high front SRV would cause carrier recombination before they reach the back junction. Hence front surface passivation is especially important for the IBC-SHJ cell. In later simulations, the front SRV value is chosen as $80 \mathrm{~cm} / \mathrm{s}$ to simulate experimental results [1], unless otherwise stated.

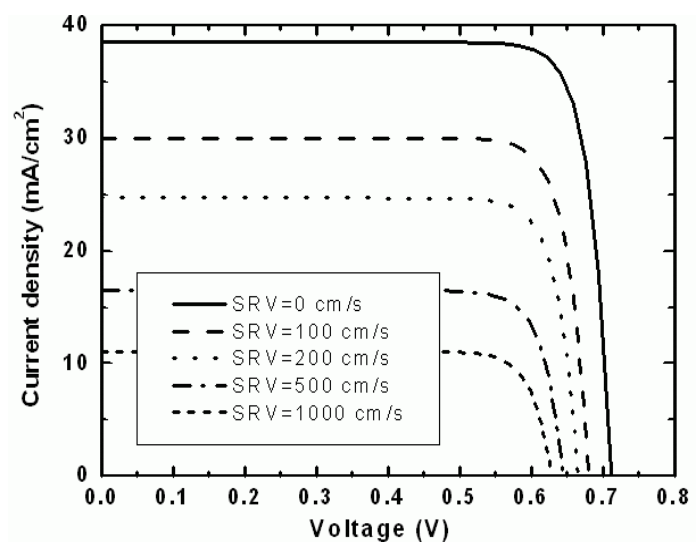

Figure 3. J-V curves for IBC-SHJ cell with different front surface recombination velocity SRV.
The effect of different $p$ and $n$ strip widths was evaluated while keeping the total device width and number of $p$ and $n$ segments fixed. The resulting $J_{s c}$ vs. n-strip width is shown in Figure 4, where the surface passivation qualities are assumed the same (front $S R V=80 \mathrm{~cm} / \mathrm{s}$, back surface ideal). As seen, as n-strip width increases, Jsc decreases, resulting in lower cell efficiency. The $V_{O C}$ and FF (not shown here) remain almost unchanged due to the assumption of constant surface passivation quality. Additionally, $\mathrm{V}_{\mathrm{OC}}$ is minimally affected by the change of $\mathrm{J}_{\mathrm{SC}}$ since both $\mathrm{J}_{\mathrm{SC}}$ and $\mathrm{J}_{\mathrm{o}}$ decrease as $\mathrm{n}$-strip width increases; i.e. $J_{0}$ reduces with decreasing emitter width.

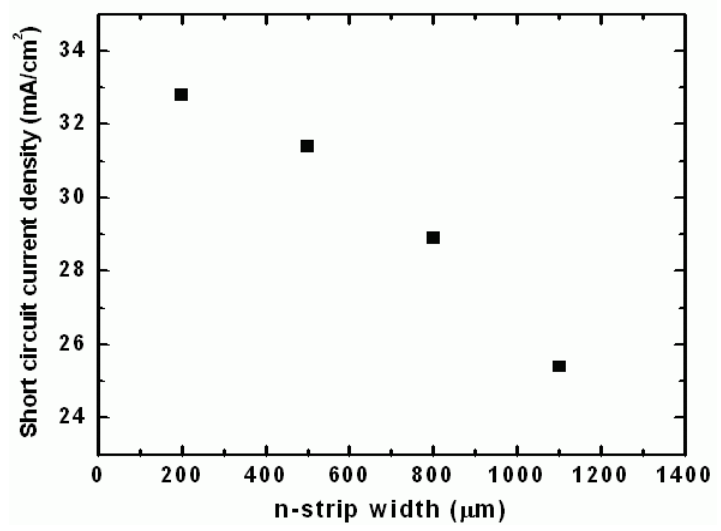

Figure 4. Jsc as function of n-strip width.

\section{Effect of strip width}

\section{LBIC line scan}


A laser beam induced current (LBIC) line scan was measured and simulated to understand the effect of strip width. In the simulation, a $50 \mu \mathrm{m}$ wide light beam (instead of laser beam) is incident on the front surface of the device, and scanned from left to right side of the device. The red dots in Figure 5 show the simulated $\mathrm{J}_{\mathrm{SC}}$ vs. position. When the light beam is over the n-strip (base contact), the current is lower compared to over the p-strip (emitter), consistent with experimental results in Ref. [9], which is also shown in the figure with the black solid line. This is because the minority carriers generated at the nstrip region need to travel not only vertically to the back junction, but also laterally to reach p-strip, the collecting junction. This longer travelling distance increases the chances for carrier recombination. So decreasing n-strip width increases the cell performance, consistent to Fig.4.

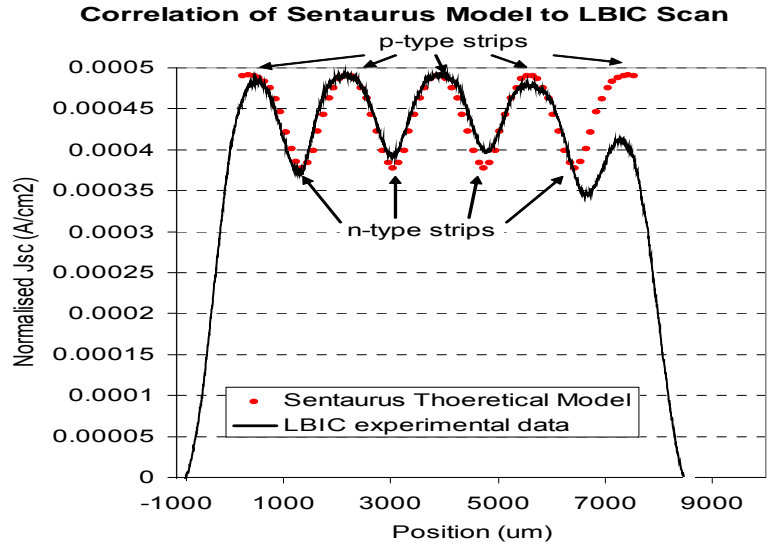

Figure 5. Simulated LBIC line scan.

\section{Improving FF for S-shape J-V}

Experimentally, deposition of an intrinsic a-Si buffer layer over the entire rear surface of the IBC-SHJ solar cell improves surface passivation, leading to a dramatic improvement of $\mathrm{V}_{\mathrm{OC}}$ and $\mathrm{J}_{\mathrm{SC}}$ [7], but a reduction in fill factor (FF), and " $S$ " shape J-V curve is often observed. Hence, simulations are also performed to guide the experiments to improve FF. It was found out from experiment that low FF with an "S" shape J-V appears only in the presence of intrinsic a-Si:H buffer layer on the $\mathrm{p}$ type emitter strip, three approaches to modify the intrinsic a-Si:H buffer are proposed here: 1) decreasing the thickness; 2) increasing the doping (conductivity); or 3) reducing the band gap. Figure 6 (a), (b) and (c) show the simulated J-V curves for varied buffer layer thickness, boron doping and band gap respectively. For each case, the other parameters (for example surface passivation quality) except the assigned variation are assumed to be the same; hence, the simulated $\mathrm{J}_{\mathrm{SC}}$ and $\mathrm{V}_{\mathrm{OC}}$ are similar. From the figures, all three methods can improve FF: (a) As the thickness of buffer layer decreases, the FF increases; and the S-shape is totally removed when buffer layer thickness decreases from $10 \mathrm{~nm}$ to $0 \mathrm{~nm}$. (b) As increasing the conductivity in the p-strip buffer layer from intrinsic to boron doping concentration of $2.2 \times 10^{18} \mathrm{~cm}^{-3}$, which is about one tenth of the doping level of p-type emitter layer $\left(2.05 \times 10^{19} \mathrm{~cm}^{-3}\right)$, FF attains a value of $\sim 78 \%$ without any "S" shape. (c) Band gap $\left(E_{g}\right)$ of intrinsic buffer layer has a large effect on FF. The FF increases from $55 \%$ to $>78 \%$ when $E_{g}$ of buffer layer reduces from $1.72 \mathrm{eV}$ to $1.65 \mathrm{eV}$. All the above improvement in FF can be explained by the modification in band alignment and band offsets [10].
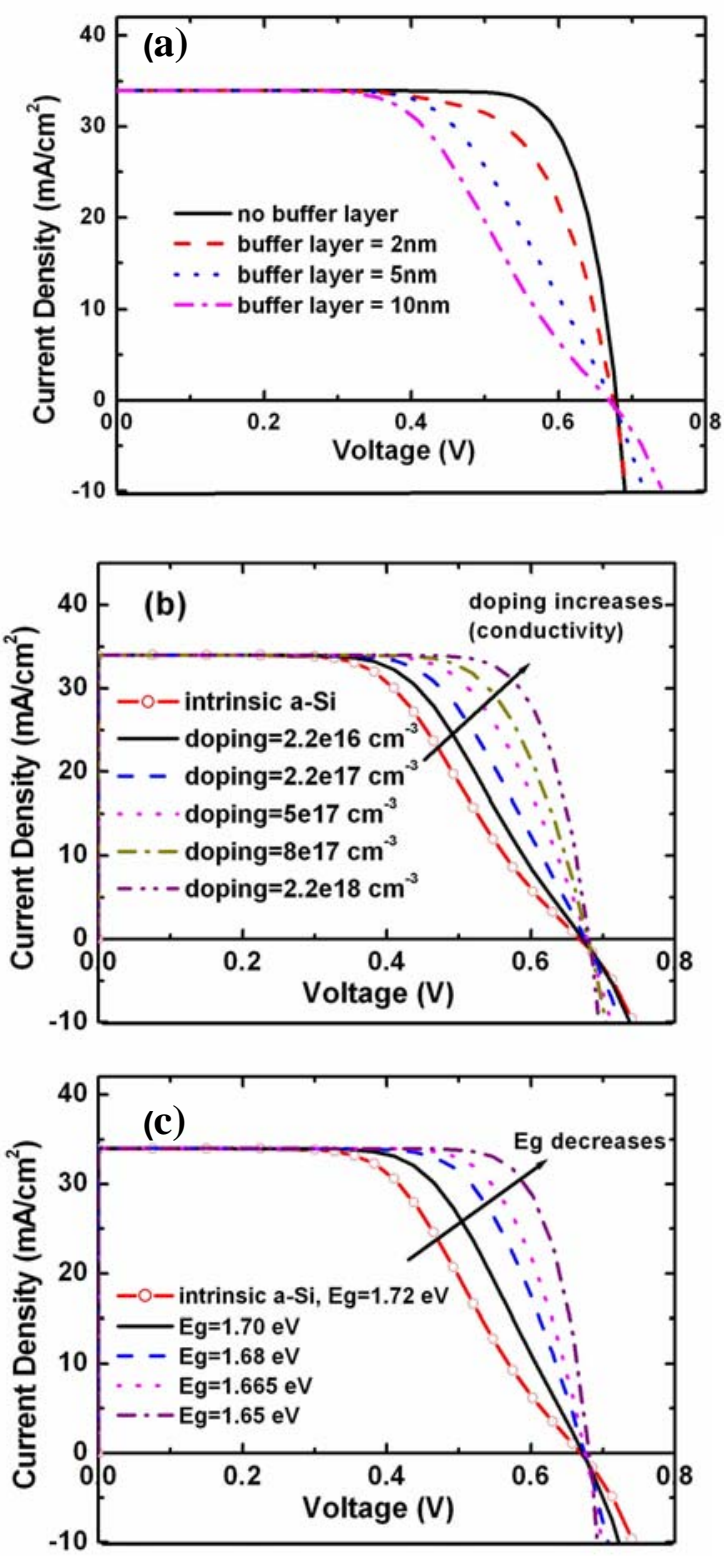

Figure 6. Simulated J-V curves for IBC-SHJ cells with different buffer layer parameters: (a) thickness, (b) boron doping (conductivity) and (c) band gap.

Efficiency potential for IBC-SHJ solar cell 
Using the present device dimensions, with a front SRV of $10 \mathrm{~cm} / \mathrm{s}$ (a value consistent with our lifetime measurements of $\sim 2$ milliseconds), an efficiency of $22.8 \%$ can be reached (Fig. 3). Further increases in efficiency will be obtained by optimizing the device dimensions. For instance, by reducing $n$-strip width to $200 \mu \mathrm{m}$ with fixed total sum of $\mathrm{p}$ - and n-strip based on Fig. 1, J $\mathrm{J}_{\mathrm{Sc}}$ can be increased and the efficiency will be enhanced to $23.8 \%$. Also the dimension of pitches, total of $p$ - plus $n$ - strips, can be optimized, where for traditional diffused IBC cells, the cell efficiency increases by $10 \%$ as pitch dimensions decreases from $1.7 \mathrm{~mm}$ to $600 \mu \mathrm{m}$ [6]. Similar trends should also be valid for IBC-SHJ solar cells. Since the junction is at back surface for IBC-SHJ cells, the thinner wafer thickness would give higher current due to less bulk carrier recombination in the wafer, but light trapping will be required. Finally, the material properties of a-Si layers used in simulation were obtained by fitting to the experiments, and might not be ideal. Further optimization to the a-Si properties should improve the cell performance. In accounting for all of the above, an efficiency of $26 \%$ is achievable for a textured IBC-SHJ solar cell.

\section{CONCLUSION}

In this paper, a two-dimensional simulation of IBC-SHJ solar cells has been established by using Sentaurus Device. The 2D nature of IBC-SHJ devices was evaluated, and current J-V curves were generated. Optimizations of IBC-SHJ solar cells were then discussed. It was shown that $\mathrm{V}_{\mathrm{OC}}$ and $\mathrm{J}_{\mathrm{SC}}$ of IBC-SHJ solar cell increase with decreasing front surface recombination velocity. The $\mathrm{J}_{\mathrm{sc}}$ improves with the increase of relative coverage of $p$-type emitter contacts, which is explained by the LBIC line scan. The S-shaped J-V curves with low fill factor (FF) observed in experiments were simulated, and three methods to improve FF by modifying the intrinsic aSi buffer layer were suggested: (i) decreased thickness, (ii) increased doping (conductivity), and (iii) reduced band gap. Finally, estimation of the potential efficiency of the IBC$\mathrm{SHJ}$ structure is addressed, and an efficiency of $26 \%$ for IBC-SHJ solar cells is potentially achievable.

\section{ACKNOWLEDGEMENTS}

The authors would like to thank Kevin Hart for film depositions. This work was partly supported by the National Renewable Energy Laboratory under subcontract \#ADJ-1-30630-12 and in part by the SETP program of DOE under award \# DE-FG36-08G018077.

This report was prepared as an account of work sponsored by an agency of the United States Government. Neither the United States Government nor any agency thereof, nor any of their employees, makes any warranty, express or implied, or assumes any legal liability or responsibility for the accuracy, completeness, or usefulness of any information, apparatus, product, or process disclosed, or represents that its use would not infringe privately owned rights. Reference herein to any specific commercial product, process, or service by trade name, trademark, manufacturer, or otherwise does not necessarily constitute or imply its endorsement, recommendation, or favoring by the United States Government or any agency thereof. The views and opinions of authors expressed herein do not necessarily state or reflect those of the United States Government or any agency thereof.

\section{REFERENCES}

[1] M. Lu, S. Bowden, U. Das, and R. Birkmire, "Interdigitated back contact silicon heterojunction solar cell and the effect of front surface passivation", Appl. Phys. Lett. 91, 2007 p. 063507.

[2] M. D. Lammert and R. J. Schwartz, "The interdigitated back contact solar cell: a silicon solar cell for use in concentrated sunlight", IEEE Trans. Electron Devices 24, 1977 p.337.

[3] M. Taguchi, K. Kawamoto, S. Tsuge, T. Baba, H. Sakata, M. Morizane, K. Uchihashi, N. Nakamura, S. Kiyama, and O. Oota, "HIT" Cells-High Efficiency Crystalline Si Cells with Novel Structure", Prog.Photovolt: Res. Appl. 8, 2000 p. 503.

[4] U.K. Das, M. Burrows, M. Lu, S. Bowden and R.W. Birkmire, "Surface passivation and heterojunction cells on Si (100) and (111) wafers using dc and rf plasma deposited Si:H thin films", Appl. Phys. Lett. 92, 2008 p. 063504.

[5] Manual for Sentaurus Device from Synopsys Inc., Version Y-2006.06.

[6] D.S.Kim, V.Meemongkolkiat, A.Ebong, B.Rounsaville, V.Upadhyaya, A.Das and A.Rohatgi, "2D-modeling and development of interdigitated back contact solar cells on low-cost substrates", Proceedings of the 4th IEEE World Conference on Photovoltaic Energy Conversion, May 712, 2006, Waikoloa, Hawaii.

[7] M. Lu, S. Bowden, U. Das, and R. Birkmire, "a-Si/c-Si heterojunction for interdigitated back contact solar cell", Proc. $22^{\text {nd }}$ European PVSEC, 2007 p. 924.

[8] R.E.I.Schropp, M.Zeeman, Amorphous and microcrystalline silicon solar cells, Kluwer Academic, Dordrecht, 1998, pp.183.

[9] M. Lu, S. Bowden, U. Das and R. Birkmire, "Interdigitated back contact silicon heterojunction (IEBCSHJ) solar cell", Proceeding MRS 2007 Spring, San Francisco, CA, April, 2007.

[10] M. Lu, U. Das, S. Bowden, S. Hegedus, R. Birkmire, "Optimization of interdigitated back contact silicon heterojunction solar cells: tailoring hetero-interface band 
structures while maintaining surface passivation", to be submitted. 